\title{
Autoimmune Gastritis
}

National Cancer Institute

\section{Source}

National Cancer Institute. Autoimmune Gastritis. NCI Thesaurus. Code C95752.

Inflammation of the body fundic mucosa of the stomach. It results from the development of autoantibodies against the parietal and chief cells. It is associated with the presence of intestinal metaplasia and an increased risk of developing gastric carcinoma. 